\title{
REVIEW
}

\section{Adaptive optical microscopy: the ongoing quest for a perfect image}

\author{
Martin J Booth ${ }^{1,2,3}$ \\ Adaptive optics is becoming a valuable tool for high resolution microscopy, providing correction for aberrations introduced by the \\ refractive index structure of specimens. This is proving particularly promising for applications that require images from deep within \\ biological tissue specimens. We review recent developments in adaptive microscopy, including methods and applications. A range of \\ advances in different microscope modalities is covered and prospects for the future are discussed.
}

Light: Science \& Applications (2014) 3, e165; doi:10.1038/lsa.2014.46; published online 25 April 2014

Keywords: aberrations; adaptive optics; microscopy

\section{INTRODUCTION}

Optical microscopes have for a long time played a vital role in biomedical research. Their ability to provide multidimensional structural and functional information about a specimen in a non-invasive manner has permitted numerous scientific advances. These microscopes rely upon high-quality optical systems to operate at the optimum resolution, which is determined by the diffraction limit of light for conventional microscopes. However, even with perfect optical components, the performance of the microscope is affected by the optical properties of the specimen. Spatial variations in refractive index introduce aberrations as the light passes through the specimen-a problem that is exacerbated when imaging deeps into tissue. These aberrations detrimentally affect the resolution and contrast of microscope images, ultimately limiting the depth at which imaging is practical. Adaptive optics $(\mathrm{AO})$ was introduced into microscopy in order to overcome this limitation. Dynamic correction elements, such as deformable mirrors (DMs) or spatial light modulators (SLMs) have been employed to compensate specimen-induced aberrations (Figure 1). New wavefront sensing and control schemes have been developed to adapt the correction elements.

The first demonstrations of adaptive optical microscopy took place in the early 2000s. In the few years that followed, there were several innovative advances that set the scene for further research. The research in this period is well covered by two review articles ${ }^{1,2}$ and a recent compiled volume, ${ }^{3}$ so this will not be reproduced here. This present article provides a review of advances in aberration correction in adaptive microscopy that have taken place in the last half-decade. During this period, a wide range of $\mathrm{AO}$ techniques have emerged, which have considerably enhanced the available toolkit. Furthermore, the benefit of correction of specimen-induced aberrations has been shown in different areas, particularly (although not exclusively) in biomedical applications. We outline these technological developments, implementations in different microscope modalities and their applications. The future possibilities for AO microscopy in enabling high-resolution and super-resolution imaging in thick tissue are discussed.

\section{APPLICATIONS OF ADAPTIVE MICROSCOPY}

The main benefit of $\mathrm{AO}$ is the compensation of specimen-induced aberrations, where image quality is compromised by the distortion of wavefronts as they propagate through the refractive index structure of a specimen. As these aberrations affect the resolution and contrast of microscope images, their correction is necessary to maintain optimum image quality. Every specimen has a different structure, so the aberrations vary among specimens and even among different regions of the same specimen. Focusing deeper into a specimen generally leads to larger amplitude aberrations with more complex structure. This phenomenon presents a particular limitation to practical focusing depth when imaging in thick tissue specimens. Indeed, AO microscopy has been demonstrated in several imaging applications where focusing deep (i.e., tens or hundreds of micrometres) into specimens is essential. At greater depths, scattering may come to dominate over aberrations-this requires alternative approaches, which are discussed at the end of this article.

One area that has attracted particular interest is neuroscience, where the detailed and efficient observation of three-dimensional neural structures deep within tissue is of the highest importance. Adaptive correction of two-photon microscopes has been demonstrated deep in brain tissue ${ }^{4-6}$ and further investigations have been made using optical coherence microscopy. ${ }^{7}$ Similar benefits have been shown in embryology, in both early stage mouse embryos ${ }^{8-10}$ and Drosophila. ${ }^{6,11,12}$ Other demonstrations with thick biological specimens have involved plant tissue, ${ }^{11}$ Caenorhabditis elegans, ${ }^{13}$ zebrafish ${ }^{14}$ and tumour spheroids. ${ }^{15}$ The benefit of adaptive aberration correction is not confined to

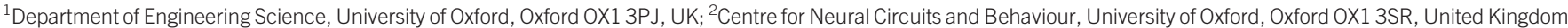
and ${ }^{3}$ School in Advanced Optical Technologies, Friedrich-Alexander Universität Erlangen-Nürnberg, 91052 Erlangen, Germany

Correspondence: Dr MJ Booth, Department of Engineering Science, University of Oxford, Parks Road, Oxford OX1 3PJ, UK

E-mail: martin.booth@eng.ox.ac.uk

Received 16 November 2013; revised 13 January 2014; accepted 13 January 2014 

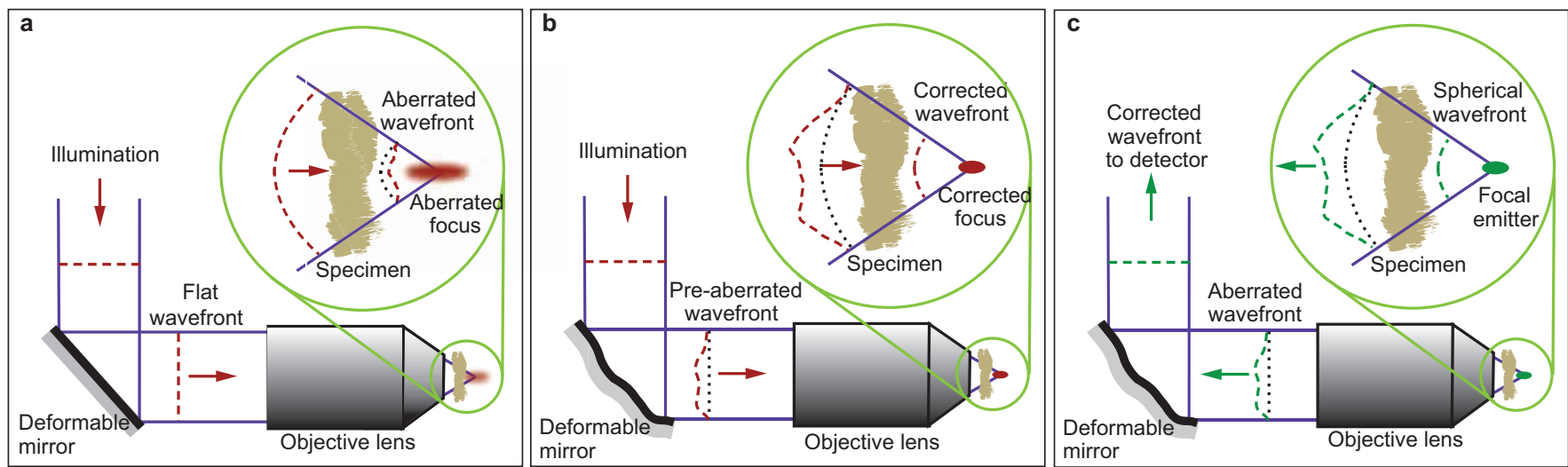

Figure 1 Principle of aberration correction in high resolution optical microscopes. (a) Aberrations are induced as light passes through the specimen due to variations in refractive index, leading to a distortion of the focus. (b) The deformable mirror introduces an aberration that cancels out the specimen induced aberration, restoring a diffraction limited focus. (c) The deformable mirror also corrects for aberrations induced in the detection or imaging path.

biological microscopy. The technology has also found application in the three-dimensional imaging of photonic structures, such as laser written waveguides in silica. ${ }^{16}$

The optics of a microscope is usually designed to operate close to the diffraction limit, but this can only be maintained over a particular range of design specifications. Some applications require operation in unusual configurations, for which the aberration tolerance of the optics might be well below the ideal. One example of this is when the wavelengths used are outside the design specification of the objective lenses. In this case, aberration correction is required in order to achieve diffraction limited performance even before focusing into a specimen. $^{10,11}$

\section{NATURE OF ABERRATIONS}

Understanding the sources of aberrations and their effects on the image formation mechanisms of a microscope is a useful starting point for the design of adaptive optical schemes. Aberrations affect not only resolution, but also image brightness and image contrast, and these effects vary according to microscope modality and the specimen structures under observation. ${ }^{17,18}$ The effects of common aberrations, such as the spherical aberration introduced by a refractive index mismatch of the lens immersion medium and specimen, had been well studied long before the first adaptive microscope implementations. Specimeninduced aberrations, arising from the structure of tissues, are more complex and a statistical description of their nature is not yet available. Observations have shown that the nature of aberrations varies across specimen types and a priori prediction of their form is not usually practical. It is this unpredictable nature that means that adaptive correction, where aberrations are both measured and corrected, is necessary to ensure optimal imaging throughout a specimen.

A current area of interest is how spatial variations in aberration affect AO microscopy. Most demonstrations of AO microscopy have made the implicit assumption that aberrations do not change over the field of view - an assumption that is often not valid. Examples of spatial aberration variations are illustrated in Figure 2. The consequence of this is that some form of 'average' correction will be made, in which some areas of the image may be improved, but others may actually be made worse. Poland et al. ${ }^{19}$ investigated how using a single correction setting compared to using a variable, point-by-point correction in brain tissue. It was shown that higher image quality could be obtained in principle by changing the aberration correction as the microscope scanned across the specimen. However, this is unlikely to be a realistic imaging mode as the bandwidth of available correction elements is much slower than the pixel rates of typical scanning microscopes. Zeng et al. ${ }^{20}$ obtained detailed three-dimensional maps of aberration variations throughout specimens and showed that this information could be used to drive region-by-region correction of aberrations - a refinement of frame averaging. Multiconjugate adaptive optics, a method already used in astronomy, has been proposed as an alternative method for dealing with spatial aberration variation. Multiconjugate adaptive optics employs multiple correction devices, which are placed in planes conjugate to different depths in the specimen. This configuration means that the correction aberration varies, in a scanning microscope, as the laser is scanned across the specimen. Simulations have shown the potential benefit of this approach, although practical implementations are still to be seen. ${ }^{21,22}$

\section{ADAPTIVE OPTICS METHODS FOR MICROSCOPY}

Recent progress in AO microscopy has been enabled by two main factors. The first factor is the commercial availability of compact deformable mirrors and spatial light modulators as adaptive correction elements. The second factor is advances in aberration measurement techniques and associated control methods. Indeed, aberration measurement and control have long been the limiting factors in the performance of AO microscopes. The past few years have seen significant developments in this area, which can be broadly categorized into direct wavefront measurement or indirect aberration optimisation. In this section we provide a brief review of the advances made in the past few years in these enabling methods.

\section{Adaptive correction elements}

Most AO microscopes have employed a DM as the adaptive correction element. The main advantages of the DM are its polarisation and wavelength-independent operation and high optical efficiency. This is well suited to use in a microscope, where the design frequently requires the use of multiple illumination and detection wavelengths. This is particularly important in fluorescence mode, where the emission is broadband and unpolarized. The various available DM models operate on different actuation principles, such as electrostatic, electromagnetic or piezoelectric, and a range of diameters and actuator numbers is available. Mirror coatings can be chosen to optimize operation at particular wavelengths or across a spectral range. Some models are available with protective windows, which are beneficial in preventing dust contamination; the delicate nature of the DMs means 

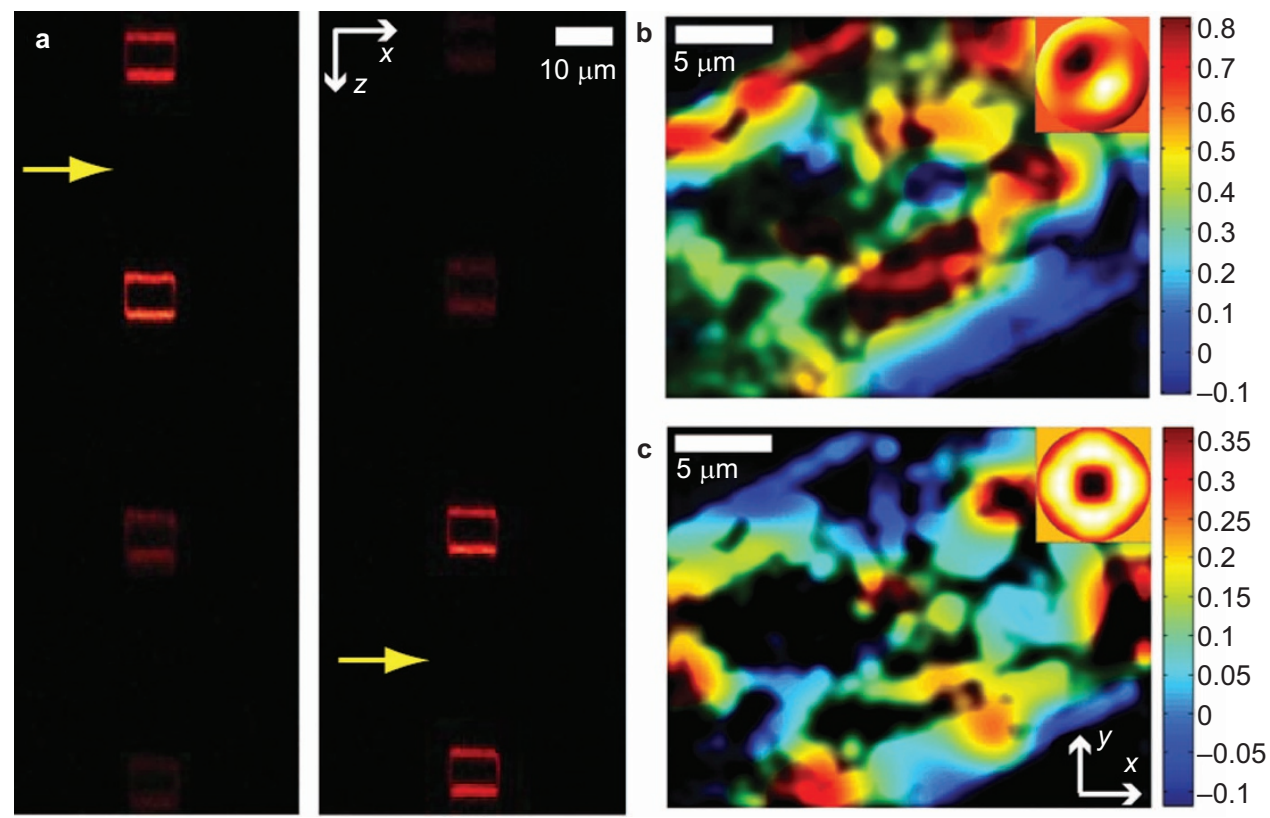

Figure 2 Spatial variation of aberrations in specimens. (a) THG microscope images show cross-sections of waveguide structures laser written at different depths in fused silica. The yellow arrow shows the two depths for which aberration correction was optimized. The signal falls significantly away from the corrected depth. (b, c) Spatial maps show the variation of two mirror deformation aberration modes (b) coma and (c) spherical across a cross-section of a Caenorhabditis elegans specimen (the mirror deformation mode amplitudes are approximately equivalent to r.m.s. phase in radians at $\lambda=1.2 \mu \mathrm{m}$ ). r.m.s., root mean square; THG, third harmonic generation.

that contact cleaning is effectively impossible without damaging the mirror surface. The choice of drive electronics and computer interface can be important in applications where fast operation is required.

The DM diameter has an influence on the microscope design, as it is important to match the pupils of the DM and the objective lens with a re-imaging system of appropriate magnification. A larger actuator number means more degrees of freedom for aberration correction. However, this does not necessarily mean that the aberration correction will be better for a particular application. For example, an actuator on a membrane mirror creates a deformation that spreads across the whole mirror surface. The deformation from each actuator therefore overlaps significantly with the deformation from all of the other actuators. On a microstructured mirror, the influence of the actuator is much more confined, meaning that there is only localized overlap between deformations from adjacent actuators. On the one hand, a membrane DM typically has a large maximum stroke and can produce large amounts of low order aberration modes, which may be advantageous for some applications. A microstructured DM, on the other hand, can typically produce more limited amplitudes of the low order modes, but with the benefit of a wider range of higher order modes.

Due to the coupling between actuators of a DM, it is usually necessary to perform a calibration or training step. This provides the information required to drive the DM with chosen aberration modes, rather than directly with individual actuator signals. A range of such methods have been developed specifically for use in microscopes, using interferometry, ${ }^{23}$ image based measurements ${ }^{24,25}$ and phase diversity. ${ }^{26,27}$

Spatial light modulators have been used less frequently in microscopes for aberration correction, due to their polarisation dependent operation and sensitivity to wavelength when used in the common modulo $2 \pi$ mode. They do, however, have many additional uses in microscopy. ${ }^{28}$ SLMs can be particularly useful in microscopes when used for beam control in the laser illumination path only. This is applicable, for example, in multiphoton microscopes. SLMs have greater flexibility than DMs in the complexity of phase patterns that they can create due to their large pixel count. As SLMs typically have a phase modulation range of around one wavelength, phase wrapping is required to reproduce larger amplitude aberrations (the modulo $2 \pi$ mode). Most SLMs are nematic liquid crystal devices that are restricted to refresh rates of at most around $100 \mathrm{~Hz}$, well below the speeds of most DMs, which are capable of several kilohertz with appropriate drivers.

\section{Direct sensing}

Direct wavefront sensing has always been a challenge in microscopy due to the complex three-dimensional nature of specimens. Wavefront sensors, such as the Shack-Hartmann sensor, are designed to operate with well-defined wavefronts from, for example, an isolated point source of light. In microscopes, light typically emanates from multiple points in the specimen simultaneously, whether from scatterers throughout the illumination cone in reflection microscopy, or from multiple fluorophores in fluorescence microscopy. The superposition of many wavefronts creates ambiguous measurements, as the desired light from the focal point is swamped by out-of-focus background. Several methods have been introduced to overcome this problem when using Shack-Hartmann sensors. Each method relies upon the exclusion of light from outside the focal region.

In-focus light can be filtered using a pinhole, much in the same way that the confocal microscope uses a pinhole to enable three-dimensional imaging. Careful choice of the pinhole diameter is required, as too large a pinhole allows too much background light through to the sensor, whereas a small pinhole would filter out any phase variation due to aberrations. This method has been implemented in scanning microscopes using backscattered light for wavefront sensing, although interpretation of the resulting measurements is not necessarily straightforward. ${ }^{29-31}$ It has been shown, for example, that sensitivity 
to different aberration modes is strongly dependent upon specimen structure and use of such sensing is likely to be reliable only as part of a carefully controlled feedback system. ${ }^{30}$

The two-photon excitation fluorescence microscope has inherent three-dimensional resolution due to the confinement of fluorescence emission to the focal region. As such, the focus provides an ideal point source for wavefront sensing. ${ }^{12,13}$ Another approach involves the use of point-like fluorescent objects as 'guide-stars' for the wavefront sensor. This can be implemented with fluorescent beads, ${ }^{6,32,33}$ although this is not compatible with many bio-imaging applications. A more sophisticated approach relies upon the labelling of sparse structures, such as centrosomes, so that an array of suitable sensing sources is available, throughout a specimen. ${ }^{6,34}$

An alternative way to avoid the effects of out-of-focus light is to use coherence gating. ${ }^{35,36}$ This method, which is particularly applicable to thick, strongly scattering specimens, such as brain tissue, uses interference of backscattered light from the specimen with a reference beam to ensure that the wavefront sensor only responds to light from the focal region. The depth selectivity is determined mainly by the coherence length of the light source, which is typically around $10 \mu \mathrm{m}$ using a superluminescent light-emitting diode.

\section{Indirect optimisation}

A common approach to aberration sensing in $\mathrm{AO}$ microscopy involves indirect measurement, whereby wavefront phase is not measured directly with a dedicated sensor, but rather via a sequence of images. These methods involve simpler hardware implementations than direct sensing systems, as they only require the addition of an adaptive element to the microscope. However, the software configuration for these systems can be more complicated.

One such indirect method is known as 'modal wavefront sensing' or 'wavefront sensorless' AO. Here the aberration is considered as a summation of orthogonal modes. A sequence of images is obtained, each with a pre-determined combination of the modes applied to the adaptive element. For each of these images, an image quality metric, such as total intensity or sharpness, is calculated and used in an optimisation process to find the optimum aberration correction. Through careful choice of the combination of modes, optimisation metric and estimator, an efficient correction scheme can be specified that permits correction of $n$ aberration modes with as few as $2 n+1$ image acquisitions independently of specimen structure. The ability to measure and correct aberrations with the fewest possible image acquisitions is important to avoid over exposure of specimens. This indirect correction method has been implemented in a range of microscope modalities. ${ }^{10,11,14,37-40}$ There are variations of this method using different combinations of measurements to enhance for example the measurement range or noise performance. ${ }^{41}$ The configuration of such system is often the most challenging task, as the algorithms rely upon the calibration of the aberration modes, which are optimally based upon the deformation modes of the deformable mirror, rather than analytic functions like the Zernike polynomials. Methods have been developed to facilitate the in situ calibration of the deformable mirror using image-based feedback. ${ }^{25}$ Further enhancements in performance could be achieved through the use of appropriate convex optimisation methods, such as quadratic programming. ${ }^{42}$

Another form of indirect sensing is based around 'pupil segmentation' ${ }^{43}$ Rather than considering the aberrated wavefront in terms of its component modes, it is instead broken down into segments. In principle, one obtains images of the specimen using one segment at a time. Each image is shifted laterally by the local tilt of the wavefront in that particular segment, so the collection of images reveals a spatially sampled approximation to the gradient of the aberration phase. From this information, it is possible to reconstruct the whole phase function, in a manner analogous to that used in the Shack-Hartmann wavefront sensor. This method has been implemented in two-photon microscopes, successfully correcting aberrations at depths over $400 \mu \mathrm{m}$ in brain tissue. ${ }^{5}$ It has also been demonstrated in wide field microscopy. ${ }^{44}$ This method has the advantage over modal sensing, in its ability to measure large amplitude aberrations, in contrast to the relatively smaller aberration range accessible to modal sensing. However, this is achieved via a greater number of image measurements. This was somewhat mitigated by the introduction of a parallelized version of the pupil segmentation approach, ${ }^{45}$ although it is interesting to note that this modification leads to, in essence, a modal sensing method.

\section{ADAPTIVE OPTICAL MICROSCOPE IMPLEMENTATIONS}

The challenges posed by aberrations are ubiquitous, wherever imaging into the bulk of specimens is necessary. It should therefore be expected that this technology has found wide applicability in a range of microscope modalities. The first adaptive microscopes were built around laser scanning systems, in particular confocal or two-photon fluorescence microscopes. These microscopes are still the major workhorses of bio-imaging laboratories, enabling three-dimensional imaging of thick tissue specimens. It is not surprising that a lot of the more recent demonstrations have also used these modalities. Applications have also progressed into other areas encompassing widefield and super-resolution microscopes and micro-endoscopy. A summary of these applications is provided here.

\section{Multiphoton microscopes}

Implementation of $\mathrm{AO}$ in a multiphoton microscope requires the placement of a correction element only in the illumination path, as aberrations affect image quality solely via this path. While aberrations also occur in the emission path, the use of a large area detector means that there is no detrimental effect on the image. Liquid crystal SLMs can readily be employed in two-photon fluorescence microscopes, as phase modulation is necessary only in the laser beam, not in the fluorescence output. ${ }^{5,43,45}$ In other two-photon systems, DMs have also been proved effective for correction of specimen-induced aberrations ${ }^{12,13,29,39}$ and laser aberrations. ${ }^{46,47} \mathrm{AO}$ has also been implemented in other multiphoton microscopes using label-free contrast based upon second harmonic generation and third harmonic generation. In these demonstrations, DMs have been used to correct aberrations only in the illumination path of the microscope, as emission path correction is not necessary. ${ }^{8-11,16}$ Some examples are illustrated in Figure 3.

\section{Confocal microscopes}

In a confocal microscope, aberration correction is required in both laser illumination and fluorescence emission paths. This is best implemented using a single DM located in a common path part of the optical train. ${ }^{6,34,48}$ It is important to note that the DM can correct both paths, despite the wavelength difference caused by the fluorescence Stokes shift. The reason for this is that the aberrations introduced by the specimen arise from the differences in optical path length through refractive index variations. The DM also introduces a correction aberration through a change in the optical path length. The changes in optical path length cancel out, so the aberration correction applies at all wavelengths simultaneously. This property holds as long as the specimen dispersion is low, which is often a reasonable approximation. The confocal microscope has also formed the basis for $\mathrm{AO}$ 

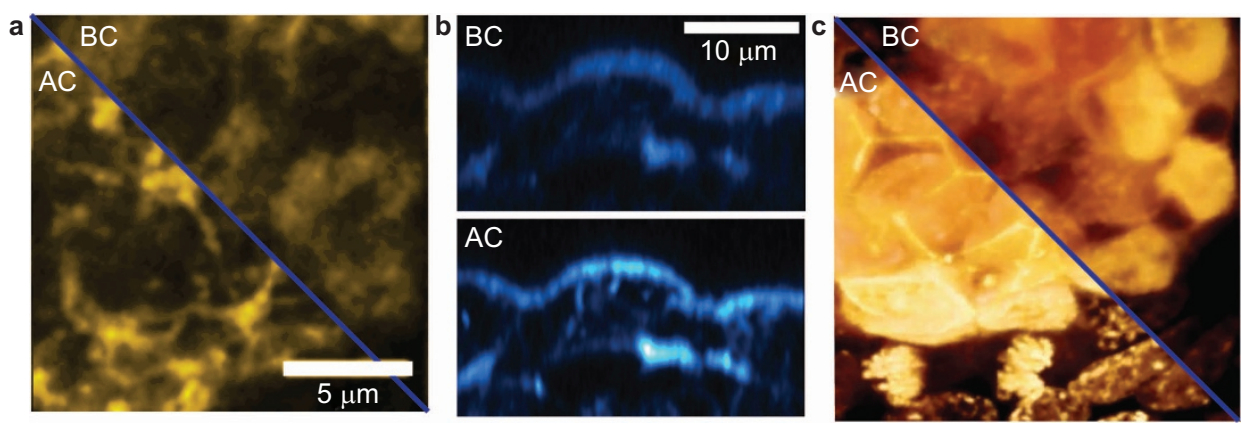

Figure 3 Demonstrations of adaptive aberration correction in multiphoton microscopes before correction and after correction. (a) Two-photon fluorescence images of GFP-labelled Drosophila fruit fly brain, lateral section. (b) Third harmonic generation microscope image of a 5.5-day-old mouse embryo, axial section. (c) Projection of $3 \mathrm{D}$ rendering of two-photon fluorescence image stack of a mouse embryo labelled with GFP and DAPI. AC, after correction; BC, before correction; 3D, threedimensional; DAPI, 4',6-diamidino-2-phenylindole; GFP, Green fluorescent protein.

fluorescence correlation spectroscopic measurements, where some measurements are particularly sensitive to the presence of aberrations. $^{49,50}$

Overall, DM-based systems might be considered more versatile for system developments, as they would be compatible with a wider range of complementary imaging modes. For example, both confocal and two-photon imaging channels could employ the same DM, whereas a SLM would be impractical in the fluorescence emission path of the confocal microscope. An exception to this might be if one were willing to sacrifice half of the emission through filtering of one polarisation component and use a SLM to modulate the remaining light.

\section{Widefield microscopes}

In widefield imaging microscopes, the light source provides flood illumination of the whole image field and aberrations in the illumination path have no effect on the image quality. Therefore, aberration correction need only be implemented in the detection path. Deformable mirrors are predominantly used in these applications as the detected light is typically broadband and randomly polarized. This is the case in transmission microscopes ${ }^{37}$ and fluorescence widefield systems, ${ }^{32,33,44,51}$ although an $\mathrm{AO}$ transmission microscope has been demonstrated using a SLM and narrowband light-emitting diode illumination. $^{52}$ Widefield imaging has also been performed in combination with adaptively corrected spatiotemporal focusing illumination. $^{53}$

\section{Structured illumination microscopes}

Widefield systems also form the basis for more complex microscope configurations. In structured illumination microscopes, widefield imaging is combined with a spatially varying illumination pattern, which can be used to perform optically sectioned, three-dimensional resolved images comparable to those obtained in a confocal microscope. In this configuration, aberrations must be corrected in both illumination and imaging paths, as both affect final image quality. ${ }^{38}$ Débarre et al. ${ }^{38}$ found that the effects of different aberration modes in these systems were considerably different than in other microscopes. Several systems have been implemented in which structured illumination is used to perform super-resolved imaging. ${ }^{54}$ It is expected that these microscopes will also benefit from adaptive aberration correction when imaging into thick specimens.

\section{Light sheet microscopes}

Light sheet microscopes (alternatively known as selective plane illumination microscopes) enable three-dimensional imaging of large (in the range of hundreds of micrometres) specimens. A conventional widefield microscope is used to image a narrow layer of the specimen, which is illuminated by a sheet of light incident from a direction perpendicular to the optic axis of the microscope. The specimen must be mounted in a suitable vessel that permits optical access from both directions. Both illumination and imaging can be affected in different ways by specimen aberrations. The predominant effect on the light sheet would be deflection away from its nominal plane. The widefield microscope would be affected by aberrations in the same way as any similar microscope. However, deflection of the light sheet would mean the illuminated layer no longer lies in the focal plane. This combination of effects means that complex adaptive compensation strategies are needed. Nevertheless, efforts have been undertaken to implement aspects of this correction in light sheet microscopes. ${ }^{14,15}$ It has also been suggested that dynamic elements could be used to control the illumination profile of a line scan illumination system for light sheet microscopy. ${ }^{55}$

\section{Micro-endoscopy}

Micro-endoscopes are attracting interest due to their potential in enabling optical biopsy for a range of clinical and research applications. The challenge in such systems is to perform high resolutionpossibly three-dimensional-imaging via a narrow endoscopes, whose dimensions constrain significantly the optical design. Graded index rod lenses offer one way to construct these endoscopes, but have rather limited flexibility, as there is essentially only one free parameter in their design-the radial refractive index profile. While on-axis optical fidelity can be optimized, there are significant aberrations as one moves to the periphery of the field of view. This contrasts with compound microscope objectives where there are multiple lens surfaces that can be shaped to optimize lens specifications, in order to minimize off axis aberrations. Adaptive optics has therefore been introduced to overcome these problems and enable high quality imaging through these imperfect optical endoscope systems. ${ }^{56-58}$ Spatial light modulators have also been used in fiber bundle endoscopes to implement beam scanning with the potential for further aberration correction. ${ }^{59}$

\section{Stimulated emission depletion (STED) microscopes}

STED microscopes create images with resolution beyond the diffraction limit by employing a combination of optical and photophysical effects. ${ }^{60,61}$ Specifically, fluorescent molecules are excited at the focus of a first laser, exactly as they would be in a confocal microscope. A second laser produces a ring-shaped focus with zero intensity at its 
centre; this depletes the excited molecules through saturated stimulated emission, leaving only a small proportion of the originally excited molecules in the vicinity of the zero intensity point. The residual fluorescence is emitted from a region much smaller than the diffraction limit of the microscope, hence the enhanced resolution. The emission is detected via the objective lens and subsequent optics by a pinhole detector, as in the confocal microscope. The ring-shaped focus is generated by the imposition of a phase mask into the optical system; in many systems this phase mask is implemented using a structured glass plate.

STED microscopes are theoretically capable of atomic level resolution, although resolutions in the order of tens of nanometres have been shown in biological specimens. Aberrations of three optical paths play a role in image formation of the STED microscope: that of the excitation beam, the depletion beam and the fluorescence detection path. For small aberrations, the quality of the depletion beam dominates the microscope performance; this has been the subject of theoretical analyses. ${ }^{62,63}$ The inclusion of an SLM in the depletion beam path has permitted correction of system aberrations,${ }^{64}$ specimen-induced aberrations ${ }^{40}$ and additionally alignment. ${ }^{65} \mathrm{~A}$ further development has permitted the generation of two overlapping depletion foci using the same SLM. ${ }^{66}$ In all of these cases, the SLM plays a dual role in providing the beam-shaping phase mask and aberration correction. As aberrations affect all three beam paths, future AO STED microscopes are likely to employ a DM that would simultaneously correct all beams.

\section{DISCUSSION}

Recent research in AO microscopy has produced a suite of tools that have enabled significant advances in the use of aberration correction. The demonstration of $\mathrm{AO}$ in a range of applications has shown the potential of this technology in improving imaging ability when focusing tens to hundreds of micrometres deep into specimens. This technology will help move microscopy into a new regime where biological studies previously confined to cell cultures and thin preparations can be performed in thick tissue and even in live specimens. There still exist, however, several challenges that must be overcome before $\mathrm{AO}$ can be a universally applicable method. Some of these challenges are discussed here.

A current aim is to develop AO systems that operate robustly and quickly for a wide selection of specimens. This will require sensing and correction schemes that can provide low wavefront error for a wide range of aberration amplitude and complexity. It is not clear that any one of the schemes described in the literature can alone provide the required flexibility. Further advances might combine both direct sensing and indirect optimisation methods. The desired increases in measurement speed and accuracy need to be achieved with minimisation of specimen exposure-an essential step for live imaging.

A further challenge is the need to cope with spatial variations in aberrations. In principle, in a scanning microscope the correction element could be adapted at high speed to follow the aberration variations, but this places significant demands on wavefront sensing. Furthermore, this would not be applicable to widefield methods, where aberrations in different field points must be corrected simultaneously. The techniques of multiconjugate adaptive optics or multiobject adaptive optics, already being developed for astronomy, may become necessary for further improvements here. ${ }^{67,68}$

The significant advances that have been made in super-resolution methods are transforming the range of biological questions that can be answered with light microscopy. STED microscopy and structured illumination microscopy have been mentioned in this article as applications where AO has facilitated deeper imaging. Single-molecule switching microscopes (such as PALM, STORM, GSDIM and their variants) ${ }^{54,60,69}$ can also benefit. Indeed, AO has been incorporated into single-molecule switching microscopes, but has not yet been demonstrated for specimen aberration correction. ${ }^{70}$ Aberrations affect the performance of these super-resolution microscopes in very different ways, as might be expected from the significant differences in image formation between the microscopes. A thorough analysis of the influence of aberrations will provide a better understanding of the principles required for $\mathrm{AO}$ control in each case.

The $\mathrm{AO}$ methods reviewed in this article have been concerned with the compensation of low-order aberrations-smooth phase variations that arise, for example, when focusing tens to hundreds of micrometres into biological tissue. These low-order aberrations are often accompanied by more complex phase variations or scattering, which are not readily corrected in an efficient manner using the same AO methods. However, significant advances have been made in phase correction for imaging through highly scattering media using optimisation procedures similar in principle to sensorless $\mathrm{AO}^{71,72}$ The complex phase variations observed in microscopy arise from the same physical phenomenon as the low order aberrations, namely, spatial variations in refractive index. In microscopy, the same specimen structure can give rise to aberration or scattering depending upon its location: a structure that produces a smooth phase variation when placed in a narrow part of the focusing cone, near the focus, could produce a complex phase variation, or scattering, when distant from the focal plane. Increasing the image depth within a specimen should therefore lead to a greater scattering contribution on top of the lower order aberrations. The development of efficient measurement and correction schemes for combined low- and high-order phase variations will be necessary to make AO practical in this imaging regime. Inspiration might be drawn from developments in astronomical 'extreme' AO where new techniques are being proposed for high-resolution, high-accuracy wavefront correction in applications such as exoplanet detection. ${ }^{67,68}$

\section{ACKNOWLEDGMENTS}

This work was supported by the following grants: EPSRC (EP/E055818/1), BBSRC (BB/J020907/1) and the Wellcome Trust (095927/A/11/Z). The images in Figures 2 and 3a were obtained by R Simmonds; in Figure $3 \mathrm{~b}$ by $\mathrm{A}$ Jesacher; and in Figure $3 \mathrm{c}$ by D Débarre.

1 Booth MJ. Adaptive optics in microscopy. Philos Trans R Soc A 2007; 365: 28292843.

2 Girkin JM, Poland SP, Wright AJ. Adaptive optics for deeper imaging of biological samples. Curr Opin Biotechnol 2009; 20: 106-110.

3 Kubby JA. Adaptive Optics for Biological Imaging. Boca Raton, FL: CRC Press; 2013.

4 Chaigneau E, Wright AJ, Poland SP, Girkin JM, Silver RA. Impact of wavefront distortion and scattering on 2-photon microscopy in mammalian brain tissue. Opt Express 2011; 19: 22755-22774.

5 Ji N, Sato TR, Betzig E. Characterization and adaptive optical correction of aberrations during in vivo imaging in the mouse cortex. Proc Natl Acad Sci USA 2012; 109: $22-27$

6 Tao X, Fernandez B, Azucena O, Fu M, Garcia D et al. Adaptive optics confocal microscopy using direct wavefront sensing. Opt Lett 2011; 36: 1062-1064.

7 Binding J, Ben Arous J, Léger JF, Gigan S, Boccara C et al. Brain refractive index measured in vivo with high-NA defocus-corrected full-field OCT and consequences for two-photon microscopy. Opt Express 2011; 19: 4833-4847.

8 Thayil A, Watanabe T, Jesacher A, Wilson T, Srinivas S et al. Long-term imaging of mouse embryos using adaptive harmonic generation microscopy. J Biomed Opt 2011; 16, 046018.

9 Watanabe T, Thayil A, Jesacher A, Grieve K, Débarre D, et al. Characterisation of the dynamic behaviour of lipid droplets in the early mouse embryo using adaptive harmonic generation microscopy. BMC Cell Biol 2010; 11: 38. 
10 Jesacher A, Thayil A, Grieve K, Debarre D, Watanabe T et al. Adaptive harmonic generation microscopy of mammalian embryos. Opt Lett 2009; 34: 3154-3156.

11 Olivier N, Débarre D, Beaurepaire E. Dynamic aberration correction for multiharmonic microscopy. Opt Lett 2009; 34: 3145-3147.

12 Tao X, Norton A, Kissel M, Azucena O, Kubby J. Adaptive optical two-photon microscopy using autofluorescent guide stars. Opt Lett 2013; 38: 5075-5078.

13 Aviles-Espinosa R, Andilla J, Porcar-Guezenec R, Olarte OE, Nieto M et al. Measurement and correction of in vivo sample aberrations employing a nonlinear guide-star in two-photon excited fluorescence microscopy. Biomed Opt Express 2011; 2: 3135-3149.

14 Bourgenot C, Saunter CD, Taylor JM, Girkin JM, Love GD. 3D adaptive optics in a light sheet microscope. Opt Express 2012; 20: 13252-13261.

15 Jorand R, Le Corre G, Andilla J, Maandhui A, Frongia C et al. Deep and clear optical imaging of thick inhomogeneous samples. PLOS ONE 2012; 7: e35795.

16 Marshall GD, Jesacher A, Thayil A, Withford MJ, Booth M. Three-dimensional imaging of direct-written photonic structures. Opt Lett 2011; 36: 695-697.

17 Simmonds RD, Wilson T, Booth MJ. Effects of aberrations and specimen structure in conventional, confocal and two-photon fluorescence microscopy. J Microsc 2012; 245: 63-71.

18 Thayil A, Jesacher A, Wilson T, Booth MJ. Influence of aberrations in third harmonic generation microscopy. J Opt 2010; 12: 084009.

19 Poland SP, Wright AJ, Cobb S, Vijverberg JC, Girkin JM. A demonstration of the effectiveness of a single aberration correction per optical slice in beam scanned optically sectioning microscopes. Micron 2011; 42: 318-323.

20 Zeng J, Mahou P, Schanne-Klein MC, Beaurepaire E, Débarre D. 3D resolved mapping of optical aberrations in thick tissues. Biomed Opt Express 2012; 3: 1898-1913.

21 Kam Z, Kner P, Agard D, Sedat JW. Modelling the application of adaptive optics to wide-field microscope live imaging. J Microsc 2007; 226: 33-42.

22 Simmonds RD, Booth MJ. Modelling of multi-conjugate adaptive optics for spatially variant aberrations in microscopy. J Opt 2013; 15: 094010.

23 Shaw M, Hall S, Knox S, Stevens R, Paterson C. Characterization of deformable mirrors for spherical aberration correction in optical sectioning microscopy. Opt Express 2010; 18: 6900-6913.

24 Turaga D, Holy TE. Image-based calibration of a deformable mirror in wide-field microscopy. Appl Opt 2010; 49: 2030-2040.

25 Thayil A, Booth MJ. Self calibration of sensorless adaptive optical microscopes. J Eur Opt Soc 2011; 6: 11045.

26 Débarre D, Vieille T, Beaurepaire E. Simple characterisation of a deformable mirror inside a high numerical aperture microscope using phase diversity. J. Microsc 2011; 244: 136-143.

27 Kromann EB, Gould TJ, Juette MF, Wilhjelm JE, Bewersdorf J. Quantitative pupi analysis in stimulated emission depletion microscopy using phase retrieval. $O p$ Lett 2012; 37: 1805-1807.

28 Maurer C, Jesacher A, Bernet S, Ritsch-Marte M. What spatial light modulators can do for optical microscopy. Laser Photon Rev 2011; 5: 81-101.

29 Cha JW, Ballesta J, So PT. Shack-Hartmann wavefront-sensor-based adaptive optics system for multiphoton microscopy. J Biomed Opt 2010; 15: 046022.

30 Rahman SA, Booth MJ. Direct wavefront sensing in adaptive optical microscopy using backscattered light. Appl Opt 2013; 52: 5523-5532.

31 Bourgenot C, Saunter CD, Love GD, Girkin JM. Comparison of closed loop and sensorless adaptive optics in widefield optical microscopy. J Eur Opt Soc 2013; 8: 13027

32 Azucena O, Crest J, Kotadia S, Sullivan W, Tao X et al. Adaptive optics wide-field microscopy using direct wavefront sensing. Opt Lett 2011; 36: 825-827.

33 Vermeulen P, Muro E, Pons T, Loriette V, Fragola A. Adaptive optics for fluorescence wide-field microscopy using spectrally independent guide star and markers. J Biomed Opt 2011; 16: 076019.

34 Tao X, Crest J, Kotadia S, Azucena O, Chen DC et al. Live imaging using adaptive optics with fluorescent protein guide-stars. Opt Express 2012; 20: 15969-15982.

35 Tuohy S, Podoleanu AG. Depth-resolved wavefront aberrations using a sensor. Opt Express 2010; 18: 3458-3476.

36 Wang J, Léger JF, Binding J, Boccara C, Gigan S et al. Measuring aberrations in the rat brain by coherence-gated wavefront sensing using a Linnik interferometer. Biomed Opt Express 2012; 3: 2510-2525.

37 Débarre D, Booth MJ, Wilson T. Image based adaptive optics through optimisation of low spatial frequencies. Opt Express 2007; 15: 8176-8190.

38 Débarre $\mathrm{D}$, Botcherby EJ, Booth MJ, Wilson T. Adaptive optics for structured illumination microscopy. Opt Express 2008; 16: 9290-9305.

39 Débarre D, Botcherby EJ, Watanabe T, Srinivas S, Booth MJ et al. Image-based adaptive optics for two-photon microscopy. Opt Lett 2009; 34: 2495-2497.

40 Gould TJ, Burke D, Bewersdorf J, Booth MJ. Adaptive optics enables 3D STED microscopy in aberrating specimens. Opt Express 2012; 20: 20998-21009.

41 Facomprez A, Beaurepaire E, Débarre D. Accuracy of correction in modal sensorless adaptive optics. Opt Express 2012; 20: 2598-2612.

42 Antonello J, Verhaegen M, Fraanje R, van Werkhoven T, Gerritsen HC et al. Semidefinite programming for model-based sensorless adaptive optics. J Opt Soc Am A 2012; 29: 2428-2438.

$43 \mathrm{Ji}$ N, Milkie DE, Betzig E. Adaptive optics via pupil segmentation for high-resolution imaging in biological tissues. Nat Methods 2010; 7: 141-147.

44 Scrimgeour J, Curtis JE. Aberration correction in wide-field fluorescence microscopy by segmented-pupil image interferometry. Opt Express 2012; 20: 14534-14541.
45 Milkie DE, Betzig E, Ji N. Pupil-segmentation-based adaptive optical microscopy with full-pupil illumination. Opt Lett 2011; 36: 4206-4208.

46 Gualda EJ, Bueno JM, Artal P. Wavefront optimized nonlinear microscopy of ex vivo human retinas. J Biomed Opt 2010; 15: 026007.

47 Bueno JM, Giakoumaki A, Gualda EJ, Schaeffel F, Artal P. Analysis of the chicken retina with an adaptive optics multiphoton microscope. Biomed Opt Express 2011; 2. 1637-1648.

48 Tao X, Azucena O, Fu M, Zuo Y, Chen DC et al. Adaptive optics microscopy with direct wavefront sensing using fluorescent protein guide stars. Opt Lett 2011; 36: 33893391.

49 Leroux CE, Wang I, Derouard J, Delon A. Adaptive optics for fluorescence correlation spectroscopy. Opt Express 2011; 19: 26839-26849.

50 Leroux CE, Grichine A, Wang I, Delon A. Correction of cell-induced optical aberrations in a fluorescence fluctuation microscope. Opt Lett 2013; 38: 2401-2403.

51 Kner P, Sedat JW, Agard DA, Kam Z. High-resolution wide-field microscopy with adaptive optics for spherical aberration correction and motionless focusing. J Microsc 2010; 237: 136-147.

52 Warber M, Maier S, Haist T, Osten W. Combination of scene-based and stochastic measurement for wide-field aberration correction in microscopic imaging. Appl Opt 2010; 49: 5474-5479.

53 Chang CY, Cheng LC, Su HW, Yen WC, Chen SJ. Widefield multiphoton microscopy with image-based adaptive optics. Proc SPIE 2012; 8520: 852000

54 Schermelleh L, Heintzmann R, Leonhardt H. A guide to super-resolution fluorescence microscopy. J Cell Biol 2010; 190: 165-175.

55 Hoda Moosavi S, Gohn-Kreuz C, Rohrbach A. Feedback phase correction of Bessel beams in confocal line light-sheet microscopy: a simulation study. Appl Opt 2013; 52: 5835-5842.

56 Bortoletto F, Bonoli C, Panizzolo P, Ciubotaru CD, Mammano F. Multiphoton fluorescence microscopy with GRIN objective aberration correction by low order adaptive optics. PLOS ONE 2011; 6: e22321.

57 Lee WM, Yun SH. Adaptive aberration correction of GRIN lenses for confocal endomicroscopy. Opt Lett 2011; 36: 4608-4610.

58 Wang C, Ji N. Pupil-segmentation-based adaptive optical correction of a highnumerical-aperture gradient refractive index lens for two-photon fluorescence endoscopy. Opt Lett 2012; 37: 2001-2003.

59 Thompson AJ, Paterson C, Neil MA, Dunsby C, French PM. Adaptive phase compensation for ultracompact laser scanning endomicroscopy. Opt Lett 2011; 36: 1707-1709.

60 Hell SW. Microscopy and its focal switch. Nat Methods 2009; 6: 24-32.

61 Eggeling C, Willig KI, Barrantes FJ. STED microscopy of living cells-new frontiers in membrane and neurobiology. J Neurochem 2013; 126: 203-212.

62 Deng S, Liu L, Cheng Y, Li R, Xu Z. Effects of primary aberrations on the fluorescence depletion patterns of STED microscopy. Opt Express 2010; 18: 1657-1666.

63 Deng S, Liu L, Cheng Y, Li R, Xu Z. Investigation of the influence of the aberration induced by a plane interface on STED microscopy. Opt Express 2009; 17, 1714 1725.

64 Auksorius E, Boruah BR, Dunsby C, Lanigan PM, Kennedy G et al. Stimulated emission depletion microscopy with a supercontinuum source and fluorescence lifetime imaging. Opt Lett 2008; 33: 113-115.

65 Gould TJ, Kromann EB, Burke D, Booth MJ, Bewersdorf J. Auto-aligning stimulated emission depletion microscope using adaptive optics. Opt Lett 2013; 38: 18601862

66 Lenz MO, Sinclair HG, Savell A, Clegg JH, Brown AC et al. 3-D stimulated emission depletion microscopy with programmable aberration correction. J Biophotonics 2014; 7: 29-36.

67 Hart M. Recent advances in astronomical adaptive optics. App/ Opt 2010; 49: D17D29.

68 Davies R, Kasper M. Adaptive optics for astronomy. Annu Rev Astron Astrophys 2012; 50: 305-351.

69 Huang B, Bates M, Zhuang X. Super resolution fluorescence microscopy. Annu Rev Biochem 2009; 78: 993-1016.

70 Izeddin I, El Beheiry M, Andilla J, Ciepielewski D, Darzacq X et al. PSF shaping using adaptive optics for three-dimensional single-molecule super-resolution imaging and tracking. Opt Express 2012; 20: 4957-4967.

71 Tang J, Germain RN, Cui M. Superpenetration optical microscopy by iterative multiphoton adaptive compensation technique. Proc Natl Acad Sci USA 2012. 109: 8434-8439.

72 Fiolka R, Si K, Cui M. Complex wavefront corrections for deep tissue focusing using low coherence backscattered light. Opt Express 2012; 20: 16532-16543.

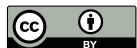

This work is licensed under a Creative Commons Attribution 3.0 Unported License. The images or other third party material in this article are included in the article's Creative Commons license, unless indicated otherwise in the credit line; if the material is not included under the Creative Commons license, users will need to obtain permission from the license holder to reproduce the material. To view a copy of this license, visit http://creativecommons.org/ licenses/by/3.0/ 